\title{
ON CONVERGENTS FORMED FROM DIOPHANTINE EQUATIONS
}

\author{
Carsten Elsner, Takao Komatsu and Iekata Shiokawa
}

University of Applied Sciences, Hannover, Germany, Hirosaki University and Keio University, Japan

ABstRaCt. We compute upper and lower bounds for the approximation of certain values $\xi$ of hyperbolic and trigonometric functions by rationals $x / y$ such that $x, y$ satisfy Diophantine equations. We show that there are infinitely many coprime integers $x, y$ such that

$$
|y \xi-x| \ll \frac{\log \log y}{\log y}
$$

and a Diophantine equation holds simultaneously relating $x, y$ and some integer $z$. Conversely, all positive integers $x, y$ with $y \geq c_{0}$ solving the Diophantine equation satisfy

$$
|y \xi-x| \gg \frac{\log \log y}{\log y}
$$

Moreover, we approximate $\sin (\pi \alpha)$ and $\cos (\pi \alpha)$ by rationals in connection with solutions of a quadratic Diophantine equation when $\tan (\pi \alpha / 2)$ is a Liouville number.

\section{Introduction AND STATEMENT of THE RESUlts In THE CASE OF HYPERBOLIC FUNCTIONS}

Let $p_{n} / q_{n}$ denote the $n$th convergent of the number

$$
e=\exp (1)=[2, \overline{1,2 k, 1}]_{k=1}^{\infty} .
$$

2000 Mathematics Subject Classification. 11D09, 11D25, 11J04, 11J70.

Key words and phrases. Diophantine approximation, Diophantine equations, trigonometric and hyperbolic functions.

The first author was supported in part by the Fund for the Promotion of International Scientific Research B-3 (2007) represented by Takao Komatsu.

The second author was supported in part by the Grant-in-Aid for Scientific Research (C) (No. 18540006), the Japan Society for the Promotion of Science. 
Put $P_{k}=p_{3 k+1}, Q_{k}=q_{3 k+1}(k=0,1,2, \ldots), P_{0}=3, P_{-1}=1, P_{-2}=1, Q_{0}=$ $1, Q_{-1}=1, Q_{-2}=-1, P_{-k}=P_{k-3}$, and $Q_{-k}=-Q_{k-3}(k=3,4,5, \ldots)$. By [5, Theorem 1.1], we know that the following identities hold.

(1.1)

$P_{n+2}=2(2 n+5) P_{n+1}+P_{n} \quad$ and $\quad Q_{n+2}=2(2 n+5) Q_{n+1}+Q_{n} \quad(n \in \mathbb{Z})$.

A similar result can be proven for the leaping convergents of the number

$$
e^{1 / s}=\exp \left(\frac{1}{s}\right)=[1, \overline{s(2 k-1)-1,1,1}]_{k=1}^{\infty} \quad(s \geq 2) .
$$

Put $P_{0}=1, P_{1}=p_{3}=2 s+1, P_{k}=p_{3 k}(k=2,3, \ldots), Q_{0}=1, Q_{1}=q_{3}=$ $2 s-1$, and $Q_{k}=q_{3 k}(k=2,3, \ldots)$. Then by [11, Theorem 1], we have

$P_{n+2}=2 s(2 n+3) P_{n+1}+P_{n} \quad$ and $\quad Q_{n+2}=2 s(2 n+3) Q_{n+1}+Q_{n} \quad(n \geq 0)$.

The preceding recurrence relations, (1.1) and (1.2), imply $P_{n} Q_{n} \equiv 1(\bmod 2)$ for all $n$ and for all $s \geq 1$. Let $h(x)$ be a function with

$$
h \in C^{(1)}[1+\delta, 3] \longrightarrow \mathbb{R}, \quad \min _{1+\delta \leq t \leq 3}\left|h^{\prime}(t)\right|>0,
$$

where $\delta$ is an arbitrary small positive number. In particular, $h^{\prime}(x)$ takes its minimum and maximum for $1+\delta \leq x \leq 3$. In our applications we choose $h(x)$ as rational functions such that at rational points $p / q$ the functions $h$ take the form

$$
h\left(\frac{p}{q}\right)=\frac{g_{1}(p, q)}{g_{2}(p, q)}
$$

where $g_{1}, g_{2} \in \mathbb{Z}[p, q]$. Then we had the following.

Lemma 1.1 ([7, Theorem 3]). Let $s \geq 1$ be an integer and let $P_{n}, Q_{n}$, and $h$ be as above. Then the inequalities

$$
C_{1} \frac{\log \log Q_{n}}{Q_{n}^{2} \log Q_{n}} \leq\left|h\left(e^{1 / s}\right)-h\left(\frac{P_{n}}{Q_{n}}\right)\right| \leq C_{2} \frac{\log \log Q_{n}}{Q_{n}^{2} \log Q_{n}} \quad(n \geq 3)
$$

hold, where $C_{1}$ and $C_{2}$ are effectively computable positive constants depending only on $s$ and the function $h$.

In [7] the application of this Lemma to various functions $h$ leads to the following approximation results. The basic idea is initiated in [6]. In what follows, all the constants $C_{3}, C_{4}, \ldots, C_{18}$ appearing in the rest of this section depend only on $s$.

Proposition 1.2. Let $s$ be a positive integer and $x$ and $y(\geq 3)$ relatively prime integers with $y \equiv 0(\bmod 2)$ such that $x^{2}+y^{2}$ is a square. Then

$$
\left|y \sinh \left(\frac{1}{s}\right)-x\right|>C_{3} \frac{\log \log y}{\log y} \text {. }
$$


On the other hand, there are infinitely many pairs $x, y$ as just described satisfying

$$
\left|y \sinh \left(\frac{1}{s}\right)-x\right|<C_{4} \frac{\log \log y}{\log y}
$$

Proposition 1.3. Let $s \geq 1$ be an integer and $x$ and $y(\geq 3)$ relatively prime integers with $y \equiv 0(\bmod 2)$ such that $x^{2}-y^{2}$ is a square. Then

$$
\left|y \cosh \left(\frac{1}{s}\right)-x\right|>C_{5} \frac{\log \log y}{\log y} .
$$

On the other hand, there are infinitely many pairs $x, y$ as just described satisfying

$$
\left|y \cosh \left(\frac{1}{s}\right)-x\right|<C_{6} \frac{\log \log y}{\log y}
$$

Proposition 1.4. Let $s \geq 1$ be an integer and $x$ and $y(\geq 3)$ relatively prime integers with $x \equiv 1(\bmod 2)$ such that $y^{2}-x^{2}$ is a square. Then

$$
\left|y \tanh \left(\frac{1}{s}\right)-x\right|>C_{7} \frac{\log \log y}{\log y} .
$$

On the other hand, there are infinitely many pairs $x, y$ as just described satisfying

$$
\left|y \tanh \left(\frac{1}{s}\right)-x\right|<C_{8} \frac{\log \log y}{\log y}
$$

\begin{tabular}{c|c|c} 
Theorem & $\begin{array}{c}\text { Diophantine } \\
\text { equation }\end{array}$ & $\xi_{s}$ \\
\hline Theorem 1.5 & $x^{2}+y^{2}=z^{4}$ & $\frac{1}{2}\left(\sinh \left(\frac{1}{s}\right)-\operatorname{cosech}\left(\frac{1}{s}\right)\right)$ \\
Theorem 1.6 & $x^{2}+y^{2}=2 z^{2}$ & $\frac{\sinh \left(\frac{1}{s}\right)-1}{\sinh \left(\frac{1}{s}\right)+1}$ \\
Theorem 1.7 & $x^{3}+4 y^{3}=z^{2}$ & $\frac{\sinh \left(\frac{4}{s}\right)+\cosh \left(\frac{4}{s}\right)+4 \sinh \left(\frac{1}{s}\right)+4 \cosh \left(\frac{1}{s}\right)}{1-2 \sinh \left(\frac{3}{s}\right)-2 \cosh \left(\frac{3}{s}\right)}$ \\
Theorem 1.8 & $x^{2}+x y+y^{2}=z^{2}$ & $\frac{2 \sinh \left(\frac{1}{s}\right)}{2+\cosh \left(\frac{1}{s}\right)-\sinh \left(\frac{1}{s}\right)}$ \\
Theorem 1.9 & $x^{2}+y^{2}=u^{4}-v^{2}$ & $\frac{1}{2} \sinh \left(\frac{2}{s}\right)$ \\
\hline
\end{tabular}

TABLE 1. Theorems dealing with hyperbolic functions 
One goal of this paper is to treat more Diophantine equations and the corresponding hyperbolic functions. A similar paper [1] appeared without giving bounds. Our emphasis here is to give computable bounds, too. We organize this paper as follows: first, we give more examples of values of hyperbolic functions, which can be approximated by rationals satisfying Diophantine equations (Theorems $1.5-1.9$ ). Then, in the final section 6 , we generalize our results to the approximation of values of trigonometric functions at specific rational points (Theorem 6.1). Finally, we treat the approximations of $\sin (\pi \alpha)$ and $\cos (\pi \alpha)$ by rationals with numerators and denominators solving the Pythagorean equation $x^{2}+y^{2}=z^{2}$, when additionally $\tan (\pi \alpha / 2)$ is assumed to be a Liouville number (Theorems 6.4, 6.5). The Table 1 gives an overview on the subsequent theorems dealing with hyperbolic functions. For any rational function $h$ let $h^{-1}$ be the inverse function, always defined in an interval centered around some $\beta$ with $h^{\prime}(\beta) \neq 0$.

THEOREM 1.5. Let $s \geq 1$ be an integer and let

$$
\xi_{s}:=\frac{1}{2}\left(\sinh \left(\frac{1}{s}\right)-\operatorname{cosech}\left(\frac{1}{s}\right)\right) .
$$

Then there are infinitely many triplets $(x, y, z)$ of integers satisfying simultaneously

$$
\left|y \xi_{s}-x\right|<C_{9} \frac{\sqrt{y} \log \log y}{\log y} \quad \text { and } \quad x^{2}+y^{2}=z^{4} .
$$

Conversely, for any integer $s \geq 1$ and for given integers $x, y(\geq 3), z$ with $x^{2}+y^{2}=z^{4}$, we have the inequality

$$
\left|y \xi_{s}-x\right|>C_{10} \frac{\sqrt{y} \log \log y}{\log y} .
$$

THEOREM 1.6. Let $s \geq 1$ be an integer and let

$$
\xi_{s}:=\frac{\sinh (1 / s)-1}{\sinh (1 / s)+1}, \quad h(t):=\frac{t^{2}-2 t-1}{t^{2}+2 t-1} .
$$

Then there are infinitely many triplets $(x, y, z)$ of integers satisfying simultaneously

$$
\left|y \xi_{s}-x\right|<C_{11} \frac{\log \log y}{\log y} \quad \text { and } \quad x^{2}+y^{2}=2 z^{2} .
$$

Conversely, for any integer $s \geq 1$ and for given positive integers $x, y(\geq 3), z$ with $y>x, h^{-1}(x / y)>\sqrt{2}-1$, and $x^{2}+y^{2}=2 z^{2}$, we have the inequality

$$
\left|y \xi_{s}-x\right|>C_{12} \frac{\log \log y}{\log y} \text {. }
$$


THEOREM 1.7. Let $s \geq 1$ be an integer and let

$$
\xi_{s}:=\frac{\sinh (4 / s)+\cosh (4 / s)+4 \sinh (1 / s)+4 \cosh (1 / s)}{1-2 \sinh (3 / s)-2 \cosh (3 / s)} .
$$

Then there are infinitely many triplets $(x, y, z)$ of integers satisfying simultaneously

$$
\left|y \xi_{s}-x\right|<C_{13} \frac{\sqrt{|y|} \log \log |y|}{\log |y|} \quad \text { and } \quad x^{3}+4 y^{3}=z^{2} .
$$

Conversely, for $s \geq 1$ and for given integers $x, y, z$ with $-y \geq 3$ and $x^{3}+4 y^{3}=$ $z^{2}$, we assume that

$$
\begin{aligned}
(x, y, z) \in\left\{\left(p\left(p^{3}+4 q^{3}\right), q\left(q^{3}-2 p^{3}\right), p^{6}-10 p^{3} q^{3}-2 q^{6}\right)\right. & : p, q \in \mathbb{Z}, \\
& \left.q\left(q^{3}-2 p^{3}\right) \leq-3\right\}
\end{aligned}
$$

and that

$$
\begin{gathered}
\frac{x}{y} \leq-\frac{(\sqrt[3]{5+3 \sqrt{3}})(3+\sqrt{3})}{3+2 \sqrt{3}} \\
\frac{p}{q}>\sqrt[3]{5+3 \sqrt{3}} \quad(s=1), \quad \sqrt[3]{1 / 2}<\frac{p}{q}<\sqrt[3]{5+3 \sqrt{3}} \quad(s>1) .
\end{gathered}
$$

Then we additionally have the inequality

$$
\left|y \xi_{s}-x\right|>C_{14} \frac{\sqrt{|y|} \log \log |y|}{\log |y|} .
$$

THEOREM 1.8. Let $s \geq 1$ be an integer and let

$$
\xi_{s}:=\frac{2 \sinh (1 / s)}{2+\cosh (1 / s)-\sinh (1 / s)} .
$$

Then there are infinitely many triplets $(x, y, z)$ of integers satisfying simultaneously

$$
\left|y \xi_{s}-x\right|<C_{15} \frac{\log \log y}{\log y} \quad \text { and } \quad x^{2}+x y+y^{2}=z^{2} .
$$

Conversely, for any integer $s \geq 1$ and for given positive integers $x, y(\geq 3), z$ with $x^{2}+x y+y^{2}=z^{2}$, we have the inequality

$$
\left|y \xi_{s}-x\right|>C_{16} \frac{\log \log y}{\log y}
$$

THEOREM 1.9. Let $s \geq 1$ be an integer and let

$$
\xi_{s}:=\frac{1}{2} \sinh \left(\frac{2}{s}\right) \text {. }
$$


Then there are infinitely many quadruplets $(x, y, u, v)$ of integers satisfying simultaneously

$$
\left|y \xi_{s}-x\right|<C_{17} \frac{\sqrt{y} \log \log y}{\log y} \quad \text { and } \quad x^{2}+y^{2}=u^{4}-v^{2} .
$$

Conversely, for any integer $s \geq 1$ and for given positive integers $x, y(\geq 3), u, v$ with $x^{2}+y^{2}=u^{4}-v^{2}$, we assume that

$$
(x, y, u, v) \in\left\{\left(p^{4}-q^{4}, 4 p^{2} q^{2}, p^{2}+q^{2}, 2 p q\left(p^{2}-q^{2}\right)\right): p, q \in \mathbb{Z}^{+}, p>q\right\} .
$$

Then we additionally have the inequality

$$
\left|y \xi_{s}-x\right|>C_{18} \frac{\sqrt{y} \log \log y}{\log y} .
$$

As can be seen from these results and their proofs, $\left|q \xi_{s}-x\right|$ tends to zero when the parametric representation of the solutions $x, y$ of the corresponding Diophantine equations are given by homogeneous forms of degree two. In Theorems 1.7, 1.9, and 6.1, it is hard to say whether all solutions of the Diophantine equations are given by the above mentioned parameterizations. Therefore, we preferred to deal with stronger conditions for the lower bounds of $\left|y \xi_{s}-x\right|$.

\section{An AUXILIARY LEMmA}

First, we mention that for every rational function

$$
R(\alpha)=\frac{g_{t} \alpha^{t}+g_{t-1} \alpha^{t-1}+\cdots+g_{0}}{h_{u} \alpha^{u}+h_{u-1} \alpha^{u-1}+\cdots+h_{0}}
$$

we have

$$
R\left(e^{1 / s}\right)=\frac{\sum_{\nu=1}^{t} g_{\nu} \sinh (\nu / s)+\sum_{\nu=0}^{t} g_{\nu} \cosh (\nu / s)}{\sum_{\mu=1}^{u} h_{\mu} \sinh (\mu / s)+\sum_{\mu=0}^{u} h_{\mu} \cosh (\mu / s)},
$$

which follows immediately from the identity $e^{1 / s}=\sinh (1 / s)+\cosh (1 / s)$. We shall apply (2.1) with integral coefficients, and $t, u$ not exceeding 4 .

Lemma 2.1. Let $s \geq 1$ be an integer and let $h(t) \in \overline{\mathbb{Q}}(t) \backslash \mathbb{Q}$. Then there exists a closed interval $I_{s}=\left[e^{1 / s}-\delta, e^{1 / s}+\delta\right]$ centered around $e^{1 / s}$ such that for any positive coprime integers $p, q$ with $q \geq 3$ the following holds.

$$
\frac{p}{q} \in I_{s} \quad \Longrightarrow \quad\left|h\left(e^{1 / s}\right)-h(p / q)\right|>C \frac{\log \log q}{q^{2} \log q},
$$

where $\delta$ and $C$ are positive constants depending possibly on $s$ and the function $h$. 
Proof. Since $h(t)$ is a non-constant rational function with algebraic coefficients, we know by Lindemann's theorem that $h\left(e^{1 / s}\right) \neq 0$ and $h^{\prime}\left(e^{1 / s}\right) \neq 0$. Hence there is a closed interval $I_{s}=\left[e^{1 / s}-\delta, e^{1 / s}+\delta\right]$ with $\delta>0$ such that

$$
h(t) \in C^{(1)}\left(I_{s}\right) \quad \text { and } \quad h^{\prime}(t) \neq 0 \quad\left(t \in I_{s}\right) .
$$

Then, by the mean-value theorem, we have

$$
C_{23}\left|e^{1 / s}-t\right|<\left|h\left(e^{1 / s}\right)-h(t)\right|<C_{24}\left|e^{1 / s}-t\right| \quad\left(t \in I_{s}\right)
$$

with

$$
C_{23}:=\min _{t \in I_{s}}\left|h^{\prime}(t)\right|>0 \quad \text { and } \quad C_{24}:=\max _{t \in I_{s}}\left|h^{\prime}(t)\right|>0 .
$$

Let $p, q$ be integers with $q \geq 3$ and $p / q \in I_{s}$. We can assume without loss of generality that

$$
\left|h\left(e^{1 / s}\right)-h(p / q)\right| \leq \frac{\log \log q}{q^{2} \log q},
$$

since otherwise for such rationals $p / q$ the inequality in (2.2) is already satisfied with $C \leq 1$. Thus, by (2.4) and (2.5), there exists a constant $C_{25}$ such that

$$
\left|e^{1 / s}-\frac{p}{q}\right|<\frac{\log \log q}{C_{23} q^{2} \log q}<\frac{1}{3 q^{2}} \quad\left(q \geq C_{25}\right) .
$$

Then from the well-known properties of the convergents of simple continued fractions, we find $p / q=p_{k} / q_{k}$ for some $k>0$, where $p_{k} / q_{k}$ is the $k$ th convergent of $e^{1 / s}$. If the $(k+1)$ th partial quotient $a_{k+1}$ of the continued fraction expansion of $e^{1 / s}$ is 1 , we have for $q \geq q_{n}$ that

$$
\left|e^{1 / s}-\frac{p}{q}\right|=\left|e^{1 / s}-\frac{p_{k}}{q_{k}}\right|>\frac{1}{\left(2+a_{k+1}\right) q_{k}^{2}}=\frac{1}{3 q_{k}^{2}} \geq \frac{1}{3 q^{2}},
$$

which contradicts (2.6). Hence, by the definition of $P_{n}$ and $Q_{n}$, i.e.,

$$
\frac{P_{n}}{Q_{n}}=\frac{p_{3 n+1}}{q_{3 n+1}} \quad(\text { if } s=1) \quad \text { or } \quad \frac{P_{n}}{Q_{n}}=\frac{p_{3 n}}{q_{3 n}} \quad(\text { if } s \geq 2),
$$

we have $p / q=P_{n} / Q_{n}$ for some $n$, and so $p=P_{n}$ and $q=Q_{n}$ since $\operatorname{gcd}(p, q)=$ 1. Therefore, by the left-hand inequality in Lemma 1.1, we get

$$
\left|h\left(e^{1 / s}\right)-h\left(\frac{p}{q}\right)\right|=\left|h\left(e^{1 / s}\right)-h\left(\frac{P_{n}}{Q_{n}}\right)\right|>C_{1} \frac{\log \log Q_{n}}{Q_{n}^{2} \log Q_{n}}=C_{1} \frac{\log \log q}{q^{2} \log q}
$$

for $q \geq C_{25}$. Hence, for some $0<C<\min \left\{1, C_{1}\right\}$, the lemma is proven.

REMARK 2.2. Lemma 2.1 also holds without the condition $\operatorname{gcd}(p, q)=1$, since then we have to deal with $Q_{n} \leq q$. 


\section{Parameter solutions of Diophantine equations}

The lemmata in this section can be proven by straightforward computations. Therefore, the details are left to the reader.

LEMMA 3.1 ([3, p.466], [4, p.256]). All positive integral solutions of

$$
x^{2}+y^{2}=z^{4}
$$

are given by

$$
\begin{aligned}
& x=\left(p^{2}-q^{2}\right)^{2}-(2 p q)^{2}=p^{4}-6 p^{2} q^{2}+q^{4}, \\
& y=4 p q\left(p^{2}-q^{2}\right), \\
& z=p^{2}+q^{2}
\end{aligned}
$$

(up to exchange of $x$ and $y$ ), where $p, q \in \mathbb{Z}$. Moreover, if we put

$$
h(t):=\frac{\left(t^{2}-1\right)^{2}-4 t^{2}}{4 t\left(t^{2}-1\right)},
$$

we have $x / y=h(p / q)$ for any solution $x, y(\neq 0)$ of the above equation (3.1). The function $h(t)$ is monotonously increasing for $t>1$, and $h \in C^{(1)}(1, \infty)$.

Lemma 3.2 ([2, p. 353, Corollary 6.3.14], [12, p. 13]). All positive integral solutions of

$$
x^{2}+y^{2}=2 z^{2}
$$

are given by

$$
\begin{aligned}
x & =p^{2}-q^{2}-2 p q, \\
y & =p^{2}-q^{2}+2 p q, \\
z & =p^{2}+q^{2}
\end{aligned}
$$

(up to exchange of $x$ and $y$ ), where $p, q \in \mathbb{Z}$. Moreover, if we put

$$
h(t):=\frac{t^{2}-2 t-1}{t^{2}+2 t-1},
$$

we have $x / y=h(p / q)$ for any solution $x, y(\neq 0)$ of the above equation (3.2). The function $h(t)$ is monotonously increasing for $t>\sqrt{2}-1$, and $h \in C^{(1)}(\sqrt{2}-1, \infty)$.

LEMMA $3.3([9])$. A set of integral solutions of

$$
x^{3}+4 y^{3}=z^{2},
$$

where $x, y, z$ are relatively prime in pairs, is given by

$$
\begin{aligned}
& x=p\left(p^{3}+4 q^{3}\right), \\
& y=q\left(q^{3}-2 p^{3}\right), \\
& z=p^{6}-10 p^{3} q^{3}-2 q^{6},
\end{aligned}
$$


where $p, q \in \mathbb{Z}$. Moreover, if we put

$$
h(t):=t \frac{4+t^{3}}{1-2 t^{3}}
$$

we have $x / y=h(p / q)$ for any solution $x, y(\neq 0)$ of the above equation (3.3). The function $h(t)$ is monotonously increasing for $0.7937 \ldots=\sqrt[3]{1 / 2}<t<$ $\sqrt[3]{5+3 \sqrt{3}}=2.1684 \ldots$, monotonously decreasing for $t>\sqrt[3]{5+3 \sqrt{3}}$, and $h \in C^{(1)}(\sqrt[3]{1 / 2}, \infty)$.

LEMMA 3.4 ([4, p. 406]). All positive integral solutions of

$$
x^{2}+x y+y^{2}=z^{2}
$$

are given by

$$
\begin{aligned}
x & =p^{2}-q^{2} \\
y & =2 p q+q^{2} \\
z & =p^{2}+p q+q^{2}
\end{aligned}
$$

(up to exchange of $x$ and $y$ ), where $p, q \in \mathbb{Z}$ with $p>q$. Moreover, if we put

$$
h(t):=\frac{t^{2}-1}{2 t+1}
$$

we have $x / y=h(p / q)$ for any solution $x, y(\neq 0)$ of the above equation (3.4). The function $h(t)$ is monotonously increasing for $t>-1 / 2$, and $h \in C^{(1)}(-1 / 2, \infty)$.

Lemma 3.5 ([4, p. 260]). A set of positive integral solutions of

$$
x^{2}+y^{2}=u^{4}-v^{2}
$$

is given by

$$
\begin{aligned}
x & =p^{4}-q^{4} \\
y & =4 p^{2} q^{2} \\
u & =p^{2}+q^{2} \\
v & =2 p q\left(p^{2}-q^{2}\right)
\end{aligned}
$$

(up to exchange of $x$ and $y$ ), where $p, q \in \mathbb{Z}$ with $p>q$. Moreover, if we put

$$
h(t):=\frac{t^{2}}{4}-\frac{1}{4 t^{2}},
$$

we have $x / y=h(p / q)$ for any solution $x, y(\neq 0)$ of the above equation (3.5). The function $h(t)$ is monotonously increasing for $t>0$, and $h \in C^{(1)}(0, \infty)$. 


\section{Proof of Theorem 1.5}

By the function $h(t)$ defined in Lemma 3.1, we have

$$
\begin{aligned}
\xi_{s} & :=h\left(e^{1 / s}\right)=\frac{\left(e^{2 / s}-1\right)^{2}-4 e^{2 / s}}{4 e^{1 / s}\left(e^{2 / s}-1\right)}=\frac{\left(e^{1 / s}-e^{-1 / s}\right)^{2}-4}{4\left(e^{1 / s}-e^{-1 / s}\right)} \\
& =\frac{\sinh ^{2}(1 / s)-1}{2 \sinh (1 / s)}=\frac{1}{2}(\sinh (1 / s)-\operatorname{cosech}(1 / s)) .
\end{aligned}
$$

Let $P_{n}, Q_{n}(n \geq 3)$ be convergents of $e^{1 / s}$ given by (2.7), and let

$$
\begin{aligned}
x_{n} & =P_{n}^{4}-6 P_{n}^{2} Q_{n}^{2}+Q_{n}^{4}, \\
y_{n} & =4 P_{n} Q_{n}\left(P_{n}^{2}-Q_{n}^{2}\right), \\
z_{n} & =P_{n}^{2}+Q_{n}^{2} .
\end{aligned}
$$

Then it follows from Lemma 3.1 and $P_{n} Q_{n} \equiv 1 \bmod 2$ that

$$
\begin{gathered}
x_{n}^{2}+y_{n}^{2}=z_{n}^{4}, x_{n}>0, y_{n}>0, z_{n}>0,4\left|x_{n}, 4\right| y_{n}, 2 \mid z_{n}, \quad \text { and } \\
x_{n} / y_{n}=h\left(P_{n} / Q_{n}\right) .
\end{gathered}
$$

Applying Lemma 1.1, we have

$$
\left|\xi_{s}-\frac{x_{n}}{y_{n}}\right|=\left|h\left(e^{1 / s}\right)-h\left(\frac{P_{n}}{Q_{n}}\right)\right| \leq C_{2} \frac{\log \log Q_{n}}{Q_{n}^{2} \log Q_{n}} .
$$

Since $1<P_{n} / Q_{n}<3$ for all integers $n \geq 1$,

$$
Q_{n}^{2}<P_{n} Q_{n}<P_{n} Q_{n}\left(P_{n}^{2}-Q_{n}^{2}\right)=\frac{y_{n}}{4}<P_{n}^{3} Q_{n}<27 Q_{n}^{4} .
$$

Particularly, for $Q_{n} \geq 3$, we get

$$
Q_{n}^{7}>\frac{Q_{n}^{3} y_{n}}{108} \geq \frac{y_{n}}{4}, \quad \log Q_{n}>\frac{\log \left(y_{n} / 4\right)}{7},
$$

and so

$$
Q_{n}^{2}>\frac{\sqrt{y_{n}}}{6 \sqrt{3}}=\frac{\sqrt{y_{n} / 4}}{3 \sqrt{3}}, \quad \log \log Q_{n}<\log \log \left(y_{n} / 4\right) .
$$

Hence, from (4.1) we conclude that

$$
\left|\xi_{s}-\frac{x_{n}}{y_{n}}\right|<21 \sqrt{3} C_{2} \frac{\log \log \left(y_{n} / 4\right)}{\sqrt{y_{n} / 4} \log \left(y_{n} / 4\right)} \quad(n \geq 1) .
$$

Setting

$$
C_{9}:=21 \sqrt{3} C_{2}, \quad x:=x_{n} / 4, y:=y_{n} / 4, z:=z_{n} / 2,
$$

we get the upper bound in Theorem 1.5. 
Conversely, we apply Lemma 2.1 to the function $h$ defined in Lemma 3.1. There exists a nontrivial closed interval $I_{s} \subset(1, \infty)$ centered around $e^{1 / s}$ such that for any positive integers $p, q(\geq 3), p / q \in I_{s}$ the inequality

$$
\left|h\left(e^{1 / s}\right)-h(p / q)\right|>C \frac{\log \log q}{q^{2} \log q}
$$

holds. Let positive integers $x, y(\geq 3), z$ be given such that $x^{2}+y^{2}=z^{4}$. Since $h((1, \infty))=\mathbb{R}, x / y$ may take every rational number. By Lemma 3.1 there are integral parameters $p, q$ with

$$
\begin{aligned}
& x=p^{4}-6 p^{2} q^{2}+q^{4}, \\
& y=4 p q\left(p^{2}-q^{2}\right), \\
& z=p^{2}+q^{2},
\end{aligned}
$$

and $x / y=h(p / q)$. By $h^{\prime}(t)>0\left(t \in I_{s}\right)$, the inverse function $h^{-1}$ exists on $h\left(I_{s}\right)$, i.e. $h^{-1}(x / y)=p / q$. Now assuming $p / q=h^{-1}(x / y) \in I_{s}$, we obtain the inequality (4.3), namely

$$
\left|\xi_{s}-\frac{x}{y}\right|>C \frac{\log \log q}{q^{2} \log q} .
$$

The interval $I_{s}$ has the form $I_{s}=\left[e^{1 / s}-\alpha, e^{1 / s}+\alpha\right]$, where $0<\alpha<e^{1 / s}-1$. Hence, if $p / q \in I_{s}$, then $p>q\left(e^{1 / s}-\alpha\right)$, so that we get $p^{2}-q^{2}>\beta q^{2}$ with $\beta:=\left(e^{1 / s}-\alpha\right)^{2}-1$. Thus we have $y=4 p q\left(p^{2}-q^{2}\right)>4 q^{4} \beta \sqrt{1+\beta}>4 \beta q^{4}$ or $q^{2}<\sqrt{y} /(2 \sqrt{\beta})$. Then, for some positive constant $C_{26}$ depending at most on $s$, we get

$$
\left|\xi_{s}-\frac{x}{y}\right|>C_{26} \frac{\log \log y}{\sqrt{y} \log y} \quad(y \geq 3) .
$$

Since $h$ is monotonously increasing on $(0, \infty)$, there exists a constant $C_{27}$ such that the inequality $\left|\xi_{s}-x / y\right|>C_{27}>0$ holds for $p / q=h^{-1}(x / y) \notin I_{s}$.

\section{Remarks on the Proofs of Theorems $1.6-1.9$}

In this section we sketch the proofs of Theorems $1.6-1.9$. The arguments are always the same as in the proof of Theorem 1.5 given in section 4 . Therefore we only mention the main formulas of the proofs.

Proof of Theorem 1.6. This Theorem is based on Lemma 3.2. Upper bound: We have

$$
\begin{aligned}
& x_{n}=P_{n}^{2}-Q_{n}^{2}-2 P_{n} Q_{n}, \\
& y_{n}=P_{n}^{2}-Q_{n}^{2}+2 P_{n} Q_{n}=\left(P_{n}+Q_{n}\right)^{2}-2 Q_{n}^{2}, \\
& z_{n}=P_{n}^{2}+Q_{n}^{2} .
\end{aligned}
$$

By $Q_{n}<P_{n}<3 Q_{n}(n \geq 1)$ we get $2 Q_{n}^{2}<y_{n}<14 Q_{n}^{2}$, and so

$$
Q_{n}^{2}>\frac{y_{n}}{14}, \quad \log Q_{n}>\frac{\log \left(y_{n} / 14\right)}{2}>C_{28} \log y_{n}, \quad \log \log Q_{n}<\log \log y_{n} .
$$


Lower bound: We have

$$
\xi_{s}=h\left(e^{1 / s}\right)=\frac{\sinh (1 / s)-1}{\sinh (1 / s)+1}
$$

and

$$
\begin{aligned}
x & =p^{2}-q^{2}-2 p q \\
y & =p^{2}-q^{2}+2 p q>3, \\
z & =p^{2}+q^{2} .
\end{aligned}
$$

The assumption $x / y<1$ of the theorem implies that $x / y$ belongs to the range of $h$. In addition, we have $I_{s} \subset[1, \infty)$. Thus, $I_{s}=\left[e^{1 / s}-\alpha, e^{1 / s}+\alpha\right]$, where $0<\alpha<e^{1 / s}-1$. If $p / q \in I_{s}$, then $p>q\left(e^{1 / s}-\alpha\right)$, and $y=(p+q)^{2}-2 q^{2}>\beta q^{2}$, where $\beta=\left(e^{1 / s}-\alpha+1\right)^{2}-2>2$. For $p / q=h^{-1}(x / y) \notin I_{s}$ and $p / q>\sqrt{2}-1$, the inequality $\left|y \xi_{s}-x\right|>C>0$ holds, since $h$ is monotonously increasing on $(\sqrt{2}-1, \infty)$. In particular, $h^{-1}$ exists for every $\varepsilon>0$ on $[\sqrt{2}-1+\varepsilon, \infty)$.

Proof of Theorem 1.7. This Theorem is based on Lemma 3.3. Upper bound: We have

$$
\begin{aligned}
& x_{n}=P_{n}\left(P_{n}^{3}+4 Q_{n}^{3}\right), \\
& y_{n}=Q_{n}\left(Q_{n}^{3}-2 P_{n}^{3}\right)<0, \\
& z_{n}=P_{n}^{6}-10 P_{n}^{3} Q_{n}^{3}-2 Q_{n}^{6} .
\end{aligned}
$$

By $Q_{n}<P_{n}<3 Q_{n}(n \geq 1)$ we get $Q_{n}^{4}<\left|y_{n}\right|<53 Q_{n}^{4}$, and so

$Q_{n}^{2}>\sqrt{\frac{\left|y_{n}\right|}{53}}, \quad \log Q_{n}>\frac{\log \left(\left|y_{n}\right| / 53\right)}{4}>C_{29} \log \left|y_{n}\right|, \quad \log \log Q_{n}<\log \log \left|y_{n}\right|$.

Lower bound: Let $\rho=\sqrt[3]{5+3 \sqrt{3}}$. By the condition of the theorem

$$
\frac{x}{y} \leq-\frac{(\sqrt[3]{5+3 \sqrt{3}})(3+\sqrt{3})}{3+2 \sqrt{3}}=h(\rho),
$$

so that $x / y(>0)$ belongs to the range of $h$. We have

$$
\begin{aligned}
\xi_{s} & =h\left(e^{1 / s}\right)=e^{1 / s} \frac{4+e^{3 / s}}{1-2 e^{3 / s}} \\
& =\frac{\sinh (4 / s)+\cosh (4 / s)+4 \sinh (1 / s)+4 \cosh (1 / s)}{1-2 \sinh (3 / s)-2 \cosh (3 / s)}<0,
\end{aligned}
$$

and

$$
\begin{aligned}
& x=p\left(p^{3}+4 q^{3}\right), \\
& y=q\left(q^{3}-2 p^{3}\right)<0, \\
& z=p^{6}-10 p^{3} q^{3}-2 q^{6} .
\end{aligned}
$$

In addition, $I_{s} \subset[\rho, \infty) \quad(s=1), \quad I_{s} \subset[1, \rho] \quad(s>1)$. Thus, $I_{s}=$ $\left[e^{1 / s}-\alpha, e^{1 / s}+\alpha\right]$, where $0<\alpha<e-\rho$ if $s=1,0<\alpha<e^{1 / s}-1$ if 
$s>1$. If $p / q \in I_{s}$, then $p>q\left(e^{1 / s}-\alpha\right)$, and $|y|=q\left(2 p^{3}-q^{3}\right)>\beta q^{4}$, where $\beta=2\left(e^{1 / s}-\alpha\right)^{3}-1>1$. If $p / q \notin I_{s}$, we again distinguish the cases $s=1$ and $s>1$. If $s=1$, we have the additional condition of the theorem that $p / q>\rho$. Since $h$ is monotonously decreasing on $(\rho, \infty)$, there exists a constant $C^{\prime}$ such that $\left|\xi_{s}-x / y\right|>C^{\prime}>0$. If $s>1$, we have $\sqrt[3]{1 / 2}<p / q<\rho$. Since $h$ is monotonously increasing on $(\sqrt[3]{1 / 2}, \rho)$, there exists a constant $C^{\prime \prime}$ such that $\left|\xi_{s}-x / y\right|>C^{\prime \prime}>0$.

Proof of Theorem 1.8. This Theorem is based on Lemma 3.4. Upper bound: We have

$$
\begin{aligned}
& x_{n}=P_{n}^{2}-Q_{n}^{2}, \\
& y_{n}=2 P_{n} Q_{n}+Q_{n}^{2}, \\
& z_{n}=P_{n}^{2}+P_{n} Q_{n}+Q_{n}^{2} .
\end{aligned}
$$

Since $Q_{n}<P_{n}<3 Q_{n}(n \geq 1)$, we have $3 Q_{n}^{2}<y_{n}<7 Q_{n}^{2}$, and so

$$
Q_{n}^{2}>\frac{y_{n}}{7}, \quad \log Q_{n}>\frac{\log \left(y_{n} / 7\right)}{2}>C_{30} \log y_{n}, \quad \log \log Q_{n}<\log \log y_{n} .
$$

Lower bound: We have

$$
\xi_{s}=h\left(e^{1 / s}\right)=\frac{2 \sinh (1 / s)}{2+\cosh (1 / s)-\sinh (1 / s)}
$$

and

$$
\begin{aligned}
x & =p^{2}-q^{2}, \\
y & =2 p q+q^{2}, \\
z & =p^{2}+p q+q^{2} .
\end{aligned}
$$

In addition, $I_{s} \subset[0, \infty)$. Hence, $I_{s}=\left[e^{1 / s}-\alpha, e^{1 / s}+\alpha\right]$, where $0<\alpha<$ $e^{1 / s}$. If $p / q \in I_{s}$, then $p>q\left(e^{1 / s}-\alpha\right)$, and $y=2 p q+q^{2}>\beta q^{2}$, where $\beta=2\left(e^{1 / s}-\alpha\right)+1>1$. Since $h$ is monotonously increasing on $[0, \infty)$, there exists a constant $C_{30}^{\prime}$ such that the inequality $\left|\xi_{s}-x / y\right|>C_{30}^{\prime}>0$ holds for $p / q=h^{-1}(x / y) \notin I_{s}$. In particular, $h^{-1}$ exists on $[0, \infty)$.

Proof of Theorem 1.9. This Theorem is based on Lemma 3.5. Upper bound: We have

$$
\begin{aligned}
x_{n} & =P_{n}^{4}-Q_{n}^{4}, \\
y_{n} & =4 P_{n}^{2} Q_{n}^{2}, \\
u_{n} & =P_{n}^{2}+Q_{n}^{2}, \\
v_{n} & =2\left(P_{n}^{2}-Q_{n}^{2}\right) P_{n} Q_{n} .
\end{aligned}
$$

Since $Q_{n}<P_{n}<3 Q_{n}(n \geq 1)$, we have $4 Q_{n}^{4}<y_{n}<36 Q_{n}^{4}$, and so

$$
Q_{n}^{2}>\frac{\sqrt{y_{n}}}{6}, \quad \log Q_{n}>\frac{\log \left(y_{n} / 36\right)}{4}>C_{31} \log y_{n}, \quad \log \log Q_{n}<\log \log y_{n} .
$$


Lower bound: We have

$$
\xi_{s}=h\left(e^{1 / s}\right)=\frac{1}{2} \sinh \left(\frac{2}{s}\right)
$$

and

$$
\begin{aligned}
x & =p^{4}-q^{4}, \\
y & =4 p^{2} q^{2}, \\
u & =p^{2}+q^{2}, \\
v & =2\left(p^{2}-q^{2}\right) p q .
\end{aligned}
$$

In addition, $I_{s} \subset(0, \infty)$, and so $I_{s}=\left[e^{1 / s}-\alpha, e^{1 / s}+\alpha\right]$, where $0<\alpha<$ $e^{1 / s}$. If $p / q \in I_{s}$, then $p>q\left(e^{1 / s}-\alpha\right)$, and $y=4 p^{2} q^{2}>\beta q^{4}$, where $\beta:=$ $4\left(e^{1 / s}-\alpha\right)^{2}>0$. Since $h$ is monotonously increasing on $(0, \infty)$, there exists a constant $C_{31}^{\prime}$ such that the inequality $\left|\xi_{s}-x / y\right|>C_{31}^{\prime}>0$ holds for $p / q=$ $h^{-1}(x / y) \notin I_{s}$. In particular, $h^{-1}$ exists for every $\varepsilon>0$ on $[\varepsilon, \infty)$.

\section{Generalization to Trigonometric Functions}

Let $s$ be a positive integer. Then, the following continued fraction expansions are known:

$$
\begin{aligned}
\tan (1) & =[1 ; \overline{2 k-1,1}]_{k=1}^{\infty}, \\
\tan (1 / s) & =[0 ; s-1, \overline{1, s-2+2 k s}]_{k=1}^{\infty} \quad(s>1) .
\end{aligned}
$$

Let $p_{n} / q_{n}$ be the $n$-th convergent of $\tan (1 / s)$. Then, we have for $P_{\nu}:=p_{2 \nu}$ and $Q_{\nu}:=q_{2 \nu}$ that

$$
\begin{aligned}
\left|\tan (1)-\frac{P_{\nu}}{Q_{\nu}}\right| & <\frac{1}{(2 \nu+1) Q_{\nu}^{2}} \quad(\nu \geq 0), \\
\left|\tan \left(\frac{1}{s}\right)-\frac{P_{\nu}}{Q_{\nu}}\right| & <\frac{1}{(s(2 \nu+1)-2) Q_{\nu}^{2}} \quad(s>1, \nu \geq 1) .
\end{aligned}
$$

Applying the method given by [8, Corollary 1], we find three-term linear recurrence formulae for $P_{n}$ and $Q_{n}$ : For $s=1$ we get with $P_{0}=1, P_{1}=$ $3, Q_{0}=1, Q_{1}=2$ that

$$
P_{n}=(2 n+1) P_{n-1}-P_{n-2}, \quad Q_{n}=(2 n+1) Q_{n-1}-Q_{n-2} \quad(n \geq 2),
$$

whereas for $s>1$ the recurrences start with $P_{0}=0, P_{1}=1, Q_{0}=1, Q_{1}=s$ :

$$
P_{n}=s(2 n-1) P_{n-1}-P_{n-2}, \quad Q_{n}=s(2 n-1) Q_{n-1}-Q_{n-2} \quad(n \geq 2) .
$$


These recurrence formulae correspond to the well-known non-regular continued fraction

$$
\tan (1 / s)=\frac{1}{s-\frac{1}{3 s-\frac{1}{5 s-\ddots}}},
$$

see $[13$, ch. $8,(27)]$. By these facts, in connection with the results for $\exp (1 / s)$ in (1.1) and (1.2), it follows that Lemma 1.1 holds for $h\left(e^{1 / s}\right)$ just as for $h(\tan (1 / s))$. As a consequence, we may replace the hyperbolic functions in our preceding results by certain trigonometric functions.

TheOREM 6.1. Let $s$ be a positive integer. Then, the theorems listed in the following table hold for the numbers $h\left(e^{1 / s}\right)$ and $h(\tan (1 / s))$ both.

\begin{tabular}{l|c|c} 
Theorem No. & $\xi_{s}=h\left(e^{1 / s}\right)$ & $\xi_{s}=h(\tan (1 / s))$ \\
\hline Proposition 1.2 & $\sinh (1 / s)$ & $-\cot (2 / s)$ \\
Proposition 1.3 & $\cosh (1 / s)$ & $\operatorname{cosec}(2 / s)$ \\
Proposition 1.4 & $\tanh (1 / s)$ & $-\cos (2 / s)$ \\
\hline Theorem 1.5 & $(\sinh (1 / s)-\operatorname{cosech}(1 / s)) / 2$ & $-\cot (4 / s)$ \\
Theorem 1.6 & $\frac{\sinh (1 / s)-1}{\sinh (1 / s)+1}$ & $\frac{1+\tan (2 / s)}{1-\tan (2 / s)}$ \\
Theorem 1.9 & $(\sinh (2 / s)) / 2$ & $-\operatorname{cosec}(2 / s) \cot (2 / s)$
\end{tabular}

A real irrational number $\xi$ is said to be a Liouville number, if there is a sequence of rationals $\left(a_{n} / b_{n}\right)_{n>0}$ with $1<b_{1}<b_{2}<\cdots$ and

$$
\left|\xi-\frac{a_{n}}{b_{n}}\right|<\frac{1}{b_{n}^{n}} \quad(n>0) .
$$

REMARK 6.2. If $\xi$ is a Liouville number and $\kappa(n)$ any strictly increasing sequence of positive integers satisfying $\kappa(n) \geq n$, then there is a sequence of rationals $\left(A_{n} / B_{n}\right)_{n>0}$ with $1<B_{1}<B_{2}<\cdots$ and

$$
\left|\xi-\frac{A_{n}}{B_{n}}\right|<\frac{1}{B_{n}^{\kappa(n)}} \quad(n>0) .
$$

This follows by setting $A_{n}:=a_{\kappa(n)}, B_{n}:=b_{\kappa(n)}(n>0)$.

REMARK 6.3. When the inequality (6.1) holds for all subscripts $n>n_{0}$ only, $\xi$ is a Liouville number defined by the shifted sequence of rationals $a_{n}^{\prime} / b_{n}^{\prime}$ with $a_{n}^{\prime}:=a_{n+n_{0}}, b_{n}^{\prime}:=b_{n+n_{0}}(n>0)$.

THEOREM 6.4. Let $\alpha$ be a real number such that $\tan (\pi \alpha / 2)$ is a Liouville number. Then there is a sequence of rationals $\left(p_{n} / q_{n}\right)_{n>0}$ with $1<q_{1}<q_{2}<$ $\cdots$ and a sequence of positive integers $\left(r_{n}\right)_{n>0}$ satisfying

$$
\left|\sin (\pi \alpha)-\frac{p_{n}}{q_{n}}\right|<\frac{1}{q_{n}^{n}}, \quad q_{n}^{2}=p_{n}^{2}+r_{n}^{2}, \quad p_{n} \equiv 0 \bmod 2 .
$$


In particular, $\sin (\pi \alpha)$ is a Liouville number.

Proof. Without loss of generality we may assume that $0<\alpha<1$. From the hypothesis of the theorem it follows that there is a sequence of rationals $\left(a_{n} / b_{n}\right)_{n>0}$ with $1<b_{1}<b_{2}<\cdots$ satisfying

$$
\left|\tan \left(\frac{\pi \alpha}{2}\right)-\frac{a_{n}}{b_{n}}\right|<\frac{1}{b_{n}^{3 n+1}} .
$$

For $0<\alpha<1$ the number $\tan (\pi \alpha / 2)$ is positive. In what follows we separate our arguments according to the cases $\tan (\pi \alpha / 2)<1$ and $\tan (\pi \alpha / 2)>1$.

CASE 1. $\tan (\pi \alpha / 2)<1$

Then, for all sufficiently large subscripts $n>n_{1}$, we have $0<a_{n} / b_{n}<1$.

CASE 2. $\tan (\pi \alpha / 2)>1$

Here, we have for large subscripts $n>n_{2}$ that $1<a_{n} / b_{n}<1+\tan (\pi \alpha / 2)$. Set

Then,

$$
f(x):=\frac{2 x}{1+x^{2}}
$$

$$
f^{\prime}(x)=\frac{2\left(1-x^{2}\right)}{\left(1+x^{2}\right)^{2}} .
$$

For $n>0$ there is a real number $\eta$ depending possibly on $\alpha$ and $n$ such that

$$
\left|f\left(\tan \left(\frac{\pi \alpha}{2}\right)\right)-f\left(\frac{a_{n}}{b_{n}}\right)\right|=\left|f^{\prime}(\eta)\right|\left|\tan \left(\frac{\pi \alpha}{2}\right)-\frac{a_{n}}{b_{n}}\right|,
$$

where either $a_{n} / b_{n}<\eta<\tan (\pi \alpha / 2)$ or $\tan (\pi \alpha / 2)<\eta<a_{n} / b_{n}$ holds. By our construction, the situation described in Case 1 yields $0<\eta<1$ for $n>n_{1}$, whereas in Case 2 we have $1<\eta<1+\tan (\pi \alpha / 2)$ for $n>n_{2}$. Hence

$$
\begin{array}{rlr}
\frac{1}{2}\left|f^{\prime}(\eta)\right| & =\frac{\left|1-\eta^{2}\right|}{\left(1+\eta^{2}\right)^{2}} \leq\left|1-\eta^{2}\right| \\
& =\left\{\begin{array}{cc}
1-\eta^{2} \leq 1 \\
\eta^{2}-1 \leq \tan (\pi \alpha / 2)(2+\tan (\pi \alpha / 2)) & \text { (Case 2) }
\end{array},\right.
\end{array}
$$

or $\left|f^{\prime}(\eta)\right| \leq C_{\alpha}:=2 \max \{1 ; \tan (\pi \alpha / 2)(2+\tan (\pi \alpha / 2))\}$. In the first case we have $0<a_{n}<b_{n}$, and in the second case $0<a_{n}<b_{n}(1+\tan (\pi \alpha / 2))$, which is summarized by $0<a_{n}<C_{\alpha} b_{n}$. Now, the right-hand side of (6.2) can be estimated as follows: Let $b_{n}>1+C_{\alpha}^{2}>C_{\alpha}$, and $n>n_{1}$ or $n>n_{2}$, respectively. Then,

$$
\begin{aligned}
\left|f^{\prime}(\eta)\right| \tan \left(\frac{\pi \alpha}{2}\right)-\frac{a_{n}}{b_{n}} \mid & \leq \frac{C_{\alpha}}{b_{n}^{3 n+1}}<\frac{1}{b_{n}^{3 n}}<\frac{1}{b_{n}^{2 n}\left(1+C_{\alpha}^{2}\right)^{n}} \\
& =\frac{1}{\left(b_{n}^{2}+C_{\alpha}^{2} b_{n}^{2}\right)^{n}}<\frac{1}{\left(b_{n}^{2}+a_{n}^{2}\right)^{n}}
\end{aligned}
$$


The left-hand side of (6.2) equals to

$$
\left|\frac{2 \tan (\pi \alpha / 2)}{1+\tan ^{2}(\pi \alpha / 2)}-\frac{2 a_{n} / b_{n}}{1+a_{n}^{2} / b_{n}^{2}}\right|=\left|\sin (\pi \alpha)-\frac{2 a_{n} b_{n}}{a_{n}^{2}+b_{n}^{2}}\right| .
$$

With $p_{n}=2 a_{n} b_{n}, q_{n}:=a_{n}^{2}+b_{n}^{2}$, and $r_{n}:=\left|a_{n}^{2}-b_{n}^{2}\right|$, we have proven for sufficiently large $n$ that

$$
\left|\sin (\pi \alpha)-\frac{p_{n}}{q_{n}}\right|<\frac{1}{q_{n}^{n}} \quad \text { with } \quad p_{n}^{2}+r_{n}^{2}=q_{n}^{2},
$$

and $\left(q_{n}\right)_{n>0}$ is a strictly increasing sequence of positive integers. To prove that $\sin (\pi \alpha)$ is a Liouville number, it suffices to show that it is not rational. But, assuming the contrary, it follows by

$$
\sin (\pi \alpha) \tan ^{2}\left(\frac{\pi \alpha}{2}\right)-2 \tan \left(\frac{\pi \alpha}{2}\right)+\sin (\pi \alpha)=0
$$

that $\tan (\pi \alpha / 2)$ is an algebraic number. But every Liouville number is transcendental, a contradiction. This completes the proof of the theorem.

Observing the identity

$$
\cos (\pi \alpha)=\frac{1-\tan ^{2}(\pi \alpha / 2)}{1+\tan ^{2}(\pi \alpha / 2)}
$$

the following result can be proven in a similar manner by applying the mean value theorem to the function $f(x)=\left(1-x^{2}\right) /\left(1+x^{2}\right)$.

THEOREM 6.5. Let $\alpha$ be a real number such that $\tan (\pi \alpha / 2)$ is a Liouville number. Then there is a sequence of rationals $\left(p_{n} / q_{n}\right)_{n>0}$ with $1<q_{1}<q_{2}<$ $\cdots$ and a sequence of positive even integers $\left(r_{n}\right)_{n>0}$ satisfying

In particular, $\cos (\pi \alpha)$ is a Liouville number.

$$
\left|\cos (\pi \alpha)-\frac{p_{n}}{q_{n}}\right|<\frac{1}{q_{n}^{n}}, \quad q_{n}^{2}=p_{n}^{2}+r_{n}^{2} .
$$

\section{REFERENCES}

[1] E. B. Burger and A. M. Pillai, On Diophantine approximation along algebraic curves, Proc. Amer. Math. Soc. 136 (2008), 11-19.

[2] H. Cohen, Number Theory, vol.I: Tools and Diophantine Equations, Springer, 2007.

[3] H. Cohen, Number Theory, vol.II: Analytic and Modern Tools, Springer, 2007.

[4] L. E. Dickson, History of the theory of numbers, vol.2, Dover Publications, 2005.

[5] C. Elsner, On arithmetic properties of the convergents of Euler's number, Colloq. Math. 79 (1999), 133-145.

[6] C. Elsner, On rational approximations by Pythagorean numbers, Fibonacci Quart. 41 (2003), 98-104.

[7] C. Elsner, T. Komatsu, and I. Shiokawa, Approximation of values of hypergeometric functions by restricted rationals, J. Théorie des Nombres de Bordeaux. 19 (2007), 387-399.

[8] C. Elsner and T. Komatsu, A recurrence formula for leaping convergents of nonregular continued fractions, Linear Algebra Appl. 428 (2008), 824-833. 
[9] C. Georgikopoulos, Rational integral solutions of the equations $x^{3}+4 y^{3}=z^{2}$ and $x^{3}+2 y^{3}=z^{2}$, Bull. Soc. Math. Gréce 24 (1948), 13-19.

[10] G. H. Hardy and E. M. Wright, An introduction to the theory of numbers, fifth edition, Clarendon Press, Oxford, 1979.

[11] T. Komatsu, Arithmetical properties of the leaping convergents of $e^{1 / s}$, Tokyo J. Math. 27 (2004), 1-12.

[12] L. J. Mordell, Diophantine equations, Academic Press, London and New York, 1969.

[13] O. Perron, Die Lehre von den Kettenbrüchen, Chelsea, New York, 1950.

\section{Elsner}

FHDW, Fachhochschule für die Wirtschaft

University of Applied Sciences

Freundallee 15, D-30173 Hannover

Germany

E-mail: carsten.elsner@fhdw.de

T. Komatsu

Graduate School of Science and Technology

Hirosaki University

Hirosaki, 036-8561

Japan

E-mail: komatsu@cc.hirosaki-u.ac.jp

I. Shiokawa

Department of Mathematics

Keio University

Yokohama, 223-8522

Japan

E-mail: shiokawa@math.keio.ac.jp

Received: 10.9.2008.

Revised: 13.1.2009. 